\title{
Philosophiques
}

\section{Le recours à l'auditoire universel implique-t-il une pétition de principe?}

\section{Guy Bouchard}

Volume 7, numéro 2, octobre 1980

URI : https://id.erudit.org/iderudit/203137ar

DOI : https://doi.org/10.7202/203137ar

Aller au sommaire du numéro

Éditeur(s)

Société de philosophie du Québec

ISSN

0316-2923 (imprimé)

1492-1391 (numérique)

Découvrir la revue

Citer cet article

Bouchard, G. (1980). Le recours à l'auditoire universel implique-t-il une pétition de principe? Philosophiques, 7(2), 161-188.

https://doi.org/10.7202/203137ar d'utilisation que vous pouvez consulter en ligne.

https://apropos.erudit.org/fr/usagers/politique-dutilisation/ 


\title{
LE RECOURS À L'AUDITOIRE UNIVERSEL IMPLIQUE-T-IL UNE PÉTITION DE PRINCIPE?
}

\author{
par Guy Bouchard
}

Pour la nouvelle rhétorique telle que la préconise Charles Perelman ${ }^{1}$, la logique, au sens large du terme, est l'étude du raisonnement en général, des moyens de preuve, quels qu'ils soient : Perelman s'oppose farouchement à ce que l'on assimile

1. Les ouvres de Perelman auxquelles nous aurons principalement recours seront représentées par les abréviations suivantes

RP : Rhétorique et philosopbie (en collaboration avec L. Olbrechts-Tyteca), Paris : P.U.F., 1952, XII-161 p.

TA: Traité de l'argumentation (en collaboration avec L. Olbrechts-Tyteca), Paris : P.U.F., 1958, 734 p. Nous utilisons la troisième édition, publiée en 1976 par les Éditions de l'Université de Bruxelles ; le contenu et la pagination de cette édition sont identiques à ceux de la première. $78 \mathrm{p}$.

CL : Cours de logique, Bruxelles: Presses Universitaires, 1961 ( $5^{c}$ éd.), 84, 81 et

JR : Justice et raison, Bruxelles : Presses Universitaires, 1963, $256 \mathrm{p}$.

ÉTA: Éléments d'une théorie de l'argumentation, Bruxelles: Presses Universitaires, $1968,67 \mathrm{p}$.

DMP : Droit, morale et philosophie, Paris: Librairie Générale de Droit et de Jurisprudence, 1976 ( $2^{\mathrm{e}}$ édition), $203 \mathrm{p}$.

CA : Le champ de largumentation, Bruxelles : Presses Universitaires, 1970, 408 p.

LJ : Logique juridique. Nouvelle rhétorique, Paris : Dalloz, 1976, 193 p.

ER : L'empire rhétorique. Rhétorique et argumentation, Paris : Vrin, 1977, $196 \mathrm{p}$.

$\mathrm{RP}, \mathrm{JR}, \mathrm{DMP}$ et CA sont des recueils d'articles. De la théorie de l'argumentation, on trouvera des exposés d'ensemble plus ou moins élaborés dans : CL, troisième fascicule, p. 75-86 ; LJ, p. 97-133 ; ÉTA, 67 p. ; ER, 196 p. ; TA, 734 p. ; les deux premiers exposés risquent de rester obscurs pour celui qui n'a pas déjà une certaine familiarité avec la nouvelle rhétorique. Par ailleurs, certaines équivalences doivent être soulignées : par exemple, la présentation de la logique formelle que contient le troisième fascicule de $\mathrm{CL}$ se retrouve dans Logique et argumentation (Bruxelles: Presses Universitaires, 1971). p. 5-72. ainsi que dans Logique et morale (Bruxelles: Presses Universitaires, 1969), p. 5-72 ; de même, Logique et argumentation reprend, de la page 87 à la page 147, le contenu de ÉTA, tandis que l'introduction de ce dernier texte se retrouve dans l'article "Une théorie philosophique de l'argumentation » (CA, p. 13-23). Mentionnons enfin que, comme l'indique son titre, LJ est une application, au domaine juridique, de la théorie de l'argumentation. Pour une application de cette même théorie au discours philosophique, cf. G. Bouchard, La nouvelle rhétorique : introduction à l'ouvre de Charles Perelman, Québec: Cahiers de l'Institut Supérieur des Sciences Humaines de l'Université Laval, 1980, 180 p. 
la logique à la seule logique formelle, et à ce que l'on réduise les moyens de preuve à ceux qu'utilisent les sciences mathématiques et les sciences expérimentales ${ }^{2}$, car cette assimilation et cette réduction entraînent l'annihilation de la philosophie ${ }^{3}$. Mais de même que le raisonnement comprend d'une part le raisonnement contraignant, c'est-à-dire la démonstration, d'autre part le raisonnement non contraignaat, c'est-à-dire l'argumentation ; de même la logique, au sens large du terme, englobe d'une part la logique formelle telle que l'entendent les modernes, d'autre part la rhétorique. La thétorique « a pour objet l'étude des techniques discursives visant à provoquer ou à accroître l'adhésion des esprits aux thèses qu'on présente à leur assentiment $»^{4}$. Elle implique l'existence d'un orateur, qui est celui qui présente l'argumentation, que ce soit oralement ou par écrit ; d'un discours, c'est-à-dire d'une argumentation; et d'un auditoire, qui est composé de tous ceux que vise l'argumentation. Celle-ci a pour but de susciter l'adhésion de l'auditoire. Or, pour obtenir cette adhésion, l'orateur doit s'adapter à son auditoire, autrement dit il doit fonder son argumentation sur des prémisses admises par celui-ci. C'est par

2. Sur les rapports entre logique, logique formelle et rhétorique, cf. TA, p. 12-19; "Logique et rhétorique " (avec L. Olbrechts-Tyteca), in RP ; "Introduction au colloque sur la théorie de la preuve ", in Revue internationale de philosophie, 27-28 (1954), p. 5.6 et 93 ; "Les cadres sociaux de l'argumentation", in CA, p. 24 et 35 ; ER, p. $15-18$; CL, fasc. 111, p. 11-12,75-76; "Philosophie, rhétorique, lieux communs", in Pbilosophe. critiques d'eux-mêmes (par A. Mercier et M. Silvas), Bern : Herbert Lang, Frankfurt/M, 1975 , p. 185

3. "Alors que pour le rationalisme classique, l'irrationnel, qu'il soit le produit des données sensibles, de l'imagination ou des préjugés, peut être éliminé grâce à l'usage des méthodes appropriées, pour les positivistes doit être traité d'irrationnel, de dépourvu de sens cognitif, tout ce qui échappe aux méthodes scientifiques, c'est-à-dire au calcul et au contrôle de l'expérience. It en résuite une diminution, allant jusqu'à l'anéantissement, du rôle de la philosophie »... (ÉTA, p. 65). Voir également: "Philosophie, rhétorique, lieux communs ", p. 183.

4. ÉTA, p. 7. Voir également: "Logique et rhétorique ", in RP, p. 1 ; "Acte et personne dans l'argumentation" (avec L. Olbrechts-Tyteca), in RP, p. 50 ; "De la preuve en philosophie ", in RP, p. 125. Dans des textes anciens, comme "Philosophies premières et philosophie régressive" (RP, p. 99), "Sociologie de la connaissance et philosophie de la connaissance "(RP, p. 139) ou "Logique et rhtitorique " (RP, p. 34), on trouve aussi une définition de la rhétorique comme "logique du préférable ", ou encore "logique des jugements de valeur"; mais Perelman a renoncé à ce type de formulation lorsqu'il s'est mis à contester le bien-fondé d'une opposition absolue entre jugements de réalité et jugements de valeur (cf., entre autres, TA, p. 680, et LJ, p. 114). Par ailleurs, la « nouvelle rhétorique "reprend à nouveaux frais la problématique de la rhétorique et de la dialectique anciennes, et en particulier aristotélicienne (cf. TA, p. 4-6 ; ER, p. 15). 
rapport à ce contexte que l'on peut comprendre en quoi consiste la pétition de principe :

La pétition de principe n'est pas une faute de logique, comme ceux qui ont oublié l'existence de la rhétorique l'ont prétendu pendant des siècles : la logique n'a jamais interdit l'usage du principe d'identité qui, en affirmant que toute proposition s'implique elle-même, aurait dû constituer la pétition de principe mise en forme, si cette dernière avait été une faute purement formelle. En fait, commettre une pétition de principe, c'est supposer admise une prémisse que l'interlocuteur conteste parce qu'elle postule, d'une façon plus ou moins implicite, une proposition qu'il s'agit de prouver. La vérité de la prémisse n'est pas en cause, mais uniquement l'adhésion de l'interlocuteur, ce qui montre bien que tout débat où l'on fait état d'une pétition de principe relève de la rhétorique, dont le but est justement de gagner l'adhésion de l'auditoire ${ }^{5}$.

Commet donc une pétition de principe l'orateur qui suppose admise par son auditoire une prémisse qui ne l'est pas effectivement. La question que nous posons est de savoir si l'orateur qui recourt à l'auditoire universel, au sens que la nouvelle rhétorique donne à ce terme, commet une telle pétition de principe. Pour y répondre, nous présenterons d'abord brièvement les divers types d'auditoire que mentionne Perelman, puis nous examinerons en détails les descriptions qu'il propose de l'auditoire universel. L'enjeu de cette question est le discours philosophique lui-même, puisque, selon Perelman :

Le philosophe, comme tel, s'adresse à la raison, c'est-àdire à l'auditoire universel, à l'ensemble de ceux qui sont considérés comme des hommes raisonnables et compétents en la matière ${ }^{6}$.

5. "De la preuve en philosophie ", in RP, p. 127-128. Voir également : TA, p. 150-153; ÉTA, p. $27-28$; Cl, fasc. 111 , p. $77-78 ; \mathrm{LJ}$, p. 116 . L'un de ces textes apporte une précision intéressante : "L'auditeur ne pourra prétendre qu'il y a vraiment pétition de principe que si la prémisse qu'il conteste n'a, en l'occurrence, aucun autre fondement que la conclusion même que l'on a voulu en tirer, et pour laquelle cette prémisse constituerait un chainon indispensable dans le raisonnement. II est extrêmement rare que cette dépendance soir suffisamment assurée pour que l'accusation soir admise sans réplique. En effet, pareille accusation implique que l'on peut, dans une argumentation, discerner avec précision non seulement si, oui ou non, l'énoncé d'une prémisse se distingue de l'énoncé de la conclusion, mais encore quelle part revient à un certain type d'arguments, et exclusivement à lui, dans le rapport " conclusion-prémisse-conclusion. "Or c'est à cause de la complexité de ce rapport que la discussion pour savoir s'il y a pétition de principe peut, en pratique, se développer. (TA, p. 151).

6. LJ, p. 122. 
La question que nous posons a donc trait aux différents types de discours et, en particulier, au discours philosophique. Si le discours philosophique s'adresse, comme le soutient Perelman, à l'auditoire universel, et si l'auditoire universel est bien tel qu'il le décrit, s'ensuit-il que chaque philosophe qui argumente commet, ou risque de commettre, une pétition de principe? La réponse à cette question devrait permettre non seulement de clarifier la conception perelmanienne de la philosophie, mais aussi de contribuer à préciser la nature du discours philosophique ainsi que son rapport avec d'autres types de discours.

\section{Les divers types d'auditoire}

L'auditoire, avons-nous dit, est composé de tous ceux que vise l'argumentation : cette définition permet d'éliminer des conceptions qui, trop liées à des critères matériels, soulèveraient des problèmes ${ }^{7}$. Par exemple, si l'on assimile l'auditoire à la personne que l'orateur interpelle nommément, comment pourra-t-on décrire l'acte du député anglais qui, s'adressant au président, cherche néanmoins à convaincre non seulement ceux qui l'écoutent, mais encore l'opinion publique de son pays ? Si l'on croit que l'auditoire se ramène à l'ensemble des personnes présentes devant l'orateur lorsqu'il prend la parole, comment décrire le comportement du chef de gouvernement qui, parlant devant l'ensemble des députés, a cependant renoncé à convaincre l'opposition et ne recherche que l'adhésion de ses propres partisans? De même, la vedette qui accorde une interview à un journaliste s'adresse non seulement à celui-ci, mais surtout à l'ensemble des lecteurs du journal. Enfin, comment déterminer avec précision l'auditoire d'un écrivain?

C'est la raison pour laquelle, il nous semble préférable de définir l'auditoire, en matière rhétorique, comme l'ensemble de ceux sur lesquels l'orateur veut influer par son argumentation. Chaque orateur pense, d'une façon plus ou moins consciente, à ceux qu'il cherche à persuader et qui constituent l'auditoire auquel s'adressent ses discours ${ }^{8}$.

7. Ce développerment se fonde sur TA, p. 24-25.

8. TA, p. 25. 
L'auditoire ainsi défini est très variable. Quantitativement, il va de l'orateur lui-même, dans la libération intime, à l'humanité entière, en passant par l'infinie variété des auditoires particuliers. Mais les auditoires

peuvent varier de mille autres manières, selon l'âge, le sexe, le tempérament, la compétence, et toute espèce de critères sociaux et politiques. Ils peuvent surtout varier selon les fonctions exercées, et plus particulièrement selon que le rôle des auditeurs est d'aboutir à une décision de quelque nature qu'elle soit, ou simplement de se former une opinion, d'acquérir une disposition à une action éventuelle et indéterminée 9 .

Mais si grande que soit la diversité des auditoires, certains d'entre eux présentent un intérêt particulier ${ }^{10}$.

Un mot, d'abord, de la délibération intime, qui présuppose qu'un individu se conçoit comme divisé au moins en deux interlocuteurs argumentant en sens opposé. Pour certains auteurs, tel Pascal, la délibération avec soi-même constitue le modèle du raisonnement sincère et honnête. Mais la psychanalyse a montré que l'on peut se tromper soi-même, et que les raisons données peuvent n'être que des rationalisations. La délibération avec soi-même ne peut donc faire fi de tous les procédés visant à gagner autrui : elle n'est qu'un cas particulier de l'accord avec les autres, elle s'apparente au dialogue.

Dans le dialogue, qui est une argumentation devant un seul auditeur, la technique d'argumentation n'est pas la même que devant un grand auditoire. Il faut connaitre les opinions et les valeurs auxquelles adhère l'interlocuteur et il faut, à chaque pas, obtenir son accord. La technique des questions et réponses permet ainsi de tester l'idée que l'on s'est formée de l'auditeur, ce qui n'est guère possible avec un plus grand auditoire. L'orateur devant tenir compte des réactions de son auditeur, celui-ci acquiert ainsi l'occasion de participer activement au débat. Cette technique peut cependant donner l'illusion que

9. "Les cadres sociaux de l'argumentation", in CA, p. 25.

10. Pour une présentation d'ensemble des divers types d'auditoire, of. : TA, p. 25-59; ÉTA, p. 21-26 ; ER, p. 27-31 ; "Logique et thétorique ", in RP, p. 19-22. Sont exclusivement consacrés au dialogue les articles « $\mathrm{La}$ méthode dialectique et le rôle de l'interlocuteur dans le dialogue " (in JR, p. 132-139), et "Dialectique et dialogue " (in CA, p. 228-235). 
l'on nage dans la vérité parce que chaque chaînon de l'argumentation a été admis : mais accord et vérité sont des notions distinctes, qu'il ne faut pas confondre. C'est pourtant cette confusion qui explique la prédilection des philosophes pour le dialogue.

Comment, lorsqu'il y a plusieurs auditeurs, peut-on gagner l'adhésion de tous ceux qu'on veut persuader? La chose sera plus facile si l'on s'adresse à un auditoire spécialisé, par exemple à un groupe de physiciens, à des syndicalistes, ou aux adeptes d'une religion. La caractéristique d'un tel auditoire, c'est qu'il est censé adhérer à certaines thèses connues, qui sont rarement remises en question, et sur lesquelles on peut se fonder pour lui faire admettre d'autres thèses. On n'a donc pas besoin, en pareil cas, de s'assurer explicitement de l'accord des auditeurs, car il est donné d'avance. Mais le recours à des thèses spécialisées sera peu efficace si les questions débattues font appel à divers aspects de la personnalité des auditeurs. Chaque auditeur, en effet, peut appartenir simultanément à divers auditoires et, en ce sens, ses réactions ne sont pas entièrement prévisibles. Aussi, en pareil cas, sera-t-il de mise de recourir à l'auditoire universel.

De l'auditoire universel, contentons-nous pour le moment de rappeler la définition que nous donnions plus haut, à savoir qu'il se compose de "l'ensemble de ceux qui sont considérés comme des hommes raisonnables et compétents en la matière $»$.

Quant à l'auditoire d'élite, c'est par rapport à l'auditoire universel qu'il faut le comprendre. Si l'orateur qui prétend s'adresser à l'auditoire universel ne réussit pas à convaincre tous ses auditeurs, il peut disqualifier les récalcitrants en les considérant comme stupides ou anormaux. Mais si le nombre et la valeur intellectuelle de ceux que l'on excommunie de la sorte risquent de rendre ridicule la procédure, on of posera à l'auditoire universel un auditoire d'élite, doué de moyens de connaissance exceptionnels et infaillibles, on en appellera aux bons, aux voyants, aux initiés, à ceux qui ont la grâce, ou encore à l'Être parfait. 
Le principe sous-jacent à la distinction entre ces divers types d'auditoire est quantitatif ${ }^{11}$. Mais l'application de ce principe entraîne une subdivision des auditoires en deux groupes, selon que le nombre des auditeurs est observable ou fictif. Le premier groupe rassemble la délibération intime, le dialogue et les auditoires spécialisés, tandis que le second groupe se compose de l'auditoire universel et de l'auditoire d'élite ; mais comme ce dernier n'est qu'un découpage au sein du premier, on peut à bon droit restreindre le second groupe à l'auditoire universel ${ }^{12}$. Celui-ci s'oppose donc à tous les autres types d'auditoire, et cette situation exceptionnelle nous incite à l'examiner d'une façon plus approfondie.

\section{L'auditoire universel}

Dans une première étape, nous ferons le bilan de l'ensemble des descriptions que propose, de l'auditoire universel, Charles Perelman. Nous examinerons ensuite les principaux problèmes que soulèvent ces descriptions.

\subsection{La définition de l'auditoire universel}

Qu'est-ce que l'auditoire universel ? Il est d'autant plus malaisé de répondre à cette question que les descriptions qu'en donne Perelman sont multiples et variables. Tentons pourtant d'isoler les principales caractéristiques de cet auditoire, quitte

11. « La diversité des auditoires est extrême. Ils peuvent varier quantitativement, allant de l'orateur lui-même, qui se dédouble dans la délibération intime, en passant par l'auditeur unique du dialogue et tous les auditoires particuliers, jusqu'à l'ensemble des êtres capables de raison, à savoir l'auditoire universel, qui n'est plus une réalité sociale concrète, mais une construction de l'orateur à partir d'éléments de son expérience». (« Les cadres sociaux de l'argumentation », in CA, p. 25.)

12. Dans La nouvelle rbétorique (Université de Neuchatel, Travaux du centre de recherches sémiologiques, 1969 , p. 22), Georges Vignaux procède à une simplification beaucoup plus radicale. Le problème qu'il soulève est de savoir si la dynamique des groupes peut s'appliquer à la relation entre l'orateur et son auditoire. Or, dit-il, Perelman et Olbrechts-Tyteca s'intéressent surtout à trois cas limites : le discours à l'humanité, à soi-même et à un seul interlocuteur, alors que la "dynamique des groupes concerne essentiellement des groupes de n personnes ". Mais, ajoute-t-il, il n'y a jamais, selon Perelman, d'auditoire entièrement passif ni d'orateur totalement actif, de sorte que l'interaction argumentative s'apparente davantage à la discussion multilatérale qu'à l'exposé ou au discours. "De plus, délibérations solitaires et argumentations visant "l'auditoire universel "sont des fictions. "Il faut donc conclure que, selon Vignaux, les seuls auditoires effectifs sont des groupes à $n$ personnes. 
à choisir, parmi les diverses formulations, celle qui nous semblera la plus appropriée ${ }^{13}$.

Le premier trait qui définit l'auditoire universel le met en rapport avec la notion de raison. La formulation la plus fréquente stipule que l'auditoire universel se compose de tous les êtres humains raisonnables (textes 1-4-5-6-9-10-14-ÉTA); on peut rattacher à cette formulation celle qui se réfere à tous les hommes adultes et normaux (TA). Selon une seconde formulation, l'auditoire universel serait constitué par tous les êtres raisonnables et compétents (textes 12-13-15-17-LJ); ou par tous les hommes normaux et compétents (texte 7 ) ; en deux autres cas cependant, il est question d'hommes raisonnables

13. Nous avons relevé vingt-deux textes pertinents à la problématique de l'auditoire universel. En voici la liste:

- TA, p. 34-46, 134-135, 148, 451,640.

- Cl, fasc. 1, p. 75-76.

- ÉTA, p. 24-26, 65-67.

- ER, p. 30-31, 154 .

- LJ, p. 107, 117-118, 122-123.

1- "Sociologie de la connaissance et philosophie de la connaissance ", in RP, p. $139-140$.

2- "Logique et thétorique" (avec L. Olbrechts-Tyteca), in RP, p. 21-22.

3- "Acte et personne dans l'argumentation " (avec L. Oibrechts-Tyteca), in RP, p. 51.

4- "De la preuve en philosophie", in RP, p. 126-131.

5- "Raison éternelle, raison historique ", in JR, p. 102-103.

6- "La règle de justice », in Dialectica, 142-143 (1960), p. 237-238 (repris dans JR).

7- "Peur-on fonder les droits de l'homme? ", in DMP, p. 72-73.

8- "De la temporalité comme caractère de l'argumentation ", in CA, p. 62-63.

9- "Les cadres sociaux de l'argumentation ", in CA, p. 25, 33.

10- "Philosophie et argumentation", in CA, p. 269-270.

11- "L'idéal de rationalité et la règle de justice ", in CA, p. 300-301.

12- "Le raisonnement juridique ", in CA, p. 368-371.

13- "Rhétorique et philosophie ", in Études philosophiques 1 (1969), p. 23-27 (repris dans CA).

14- "How Do We Apply Reason to Values?", in The Journal of Philosophy, L.11 (1955) 26, p. 800

15- "Philosophie, thétorique, lieux communs", Art. cité (cf. note 2), p. 187. 193.

16- "Philosophie et méthode ", Bruxelles: Éditions de l'Université, 1974, p. 193-196.

17- "Philosophie et rhétorique ", in Tijdscbrift voor Filosofie, 41 (1979) 3, p. $441-442$.

Nous nous référons aux volumes à l'aide des abréviations proposées antérieurement, et aux arricles à l'aide des chiffres que nous venons de leur arrribuer, afin de ne pas répéter inutilement les titres. It nous semble indispensable en effet de fournir, pour chaque élément de la description, l'ensemble des références pertinentes, et ce, non seulement pour permettre au lecteur de retrouver le texte de Perelman dans l'un ou l'autre des documents cités, mais aussi à titre d'indice de fréquence des diverses formulations. 
(normaux) ou compétents (textes 11-TA). Enfin, deux formulations s'écartent de la terminologie des précédentes. La première prétend que la rationalité est liée à des affirmations et à des croyances qui sont universalisables aux yeux de tous (texte 16). La seconde comporte trois volets : a) l'auditoire universel se compose de tous ceux qui sont disposés à entendre le philosophe et qui sont capables de suivre son argumentation ; b) le philosophe doit utiliser des prémisses qui sont censées s'imposer à tout être de raison suffisamment éclairé ; c) il doit recourir à des prémisses et à des arguments universalisables, c'est-à-dire acceptables en principe par tous les membres de l'auditoire universel (ER). Cette formule se rattache manifestement à la conception qui allie raison et compétence ; en même temps, elle nous permet d'interpréter la formule précédente: les prémisses universalisables, ce sont celles qui s'adressent à l'auditoire universel. On peut donc conclure que l'ensemble des textes se partage en deux groupes à peu près égaux véhiculant deux conceptions de l'auditoire universel : celui-ci se composerait, dans un cas, de tous les êtres humains raisonnables et, dans le second, de tous les êtres humains raisonnables et compétents. À cette double conception de la composition de l'auditoire universel se greffent quelques idées importantes : s'adresser à un tel auditoire, c'est s'adresser à la raison (textes 13-15), et cela oblige l'orateur à n'utiliser que des prémisses et des arguments qui se prétendent objectifs, rationnels, raisonnables, universalisables (textes 2-16-ÉTAER) ; inversement, l'argumentation adressée à l'auditoire universel est objective, rationnelle ou raisonnable ${ }^{14}$ (textes 1-7-11-14); dès lors :

celui qui a souci de l'adhésion de l'auditoire universel renoncera, même devant un auditoire particulier, à des arguments inadmissibles pour cet auditoire universel, tel qu'il se le représente ; il jugera presque immoral de recourir à une

14. À propos du symbole, Perelman écrit : "Les symboles exercent une action indéniable sur ceux qui reconnaissent la liaison symbolique, mais n'en ont aucune sur les autres : ils sont caractéristiques d'une culture particulière, mais ne peuvent servir pour l'auditoire universel, ce qui confirme leur aspect irrationnel " (TA, p. 451). Si l'auditoire universel incarne la raison, et si toute argumentation qui ne s'adresse pas à cet auditoire doit être considérée comme irrationnelle, faudra-t-il admettre que toute argumentation adressée à un auditoire concret est irrationnelle? Les philosophes auraient-ils le monopole de la rationalité ? 
argumentation qui, à ses propres yeux, ne serait pas rationnelle ${ }^{15}$.

Le second caractère de l'auditoire universel est lié à son statut ontologique. Cet auditoire n'est jamais donné effectivement, il ne s'agit pas d'une réalité sociale concrète (textes 2-3-9-10-TA), mais d'une construction mentale ${ }^{16}$ de l'orateur lui-même (textes 2-3-4-5-8-9-10-12-13-TA-ÉTA). La raison qu'incarne l'auditoire universel n'est donc pas une raison éternelle, mais une raison historique : chaque individu, chaque milieu, chaque culture, chaque époque a sa propre conception de l'auditoire universel et peut être caractérisé par elle ${ }^{17}$ (textes 1-2-3-4-5-8-13-17-TA-ÉTA-LJ). Mais si l'auditoire universel est une construction mentale variant d'un orateur à l'autre, ne risque-t-il pas d'être complètement arbitraire? Pour résoudre ce problème, Perelman souligne d'abord que l'auditoire universel a un fondement réel : l'orateur part de son expérience, de ce qu'il sait lui-même et de ce qu'il pense que les autres savent et admettent, et il s'efforce de transcender les divergences d'opinions et les oppositions dont il a conscience (textes 1-2-3-4-5-8-9-10-14-21-TA-ÉTA). C'est à partir de cette expérience que, par extrapolation, il se fait une idée de ce que tous les êtres raisonnables devraient admettre (textes 8-14-15TA-ÉTA-LJ). "L'accord d'un auditoire universel n'est donc pas une question de fait, mais de droit $^{18} »$ :
À mes yeux, dit Perelman, le discours philosophique s'inspire, dans le champ de l'argumentation, de l'impératif catégorique de Kant : le philosophe doit argumenter de façon que son discours puisse obtenir, selon lui, l'adhésion de l'auditoire universel ${ }^{19}$.

Mais cette adhésion est un souhait, une hypothèse : l'orateur doit-il se contenter de ce souhait, ou au contraire tentera-t-il de "vérifier " son hypothèse ? La position de Perelman, à ce sujet, est moins claire. Que l'orateur ne puisse jamais s'assurer de l'adhésion effective de tous les membres de l'auditoire

15. TA, p. 640.

16. L'expression "construction mentale " englobe ici toute une constellation de termes employés par Perelman : auditoire idéal, représentation que se fait l'orateur, image, produit de l'imagination, fiction, création, modèle, extrapolarion, hypothèse.

17. Cette tâche relève de la sociologie de la connaissance : cf. texte 4 .

18. TA, p. 41.

19. Texte 15, p. 188. 
universel (texte 4), cela va de soi. Mais doit-il, une fois son hypothèse construite, se croiser les bras et attendre les résultats, comme le laisse entendre le Traité de l'argumentation?

Chaque homme croit en un ensemble de faits, de vérités, que tout homme « normal » doit, selon lui, admetre, parce qu'ils sont valables pour tout être raisonnable. Mais en est-il vraiment ainsi ? Cette prétention à une validité absolue pour tout auditoire composé d'êtres raisonnables n'est-elle pas exorbitante? Même l'auteur le plus consciencieux ne peut, sur ce point, que se soumettre à l'épreuve des faits, au jugement de ses lecteurs. Il aura, en tout cas, fait ce qui dépend de lui pour convaincre, s'il croit s'adresser valablement à pareil auditoire ${ }^{20}$.

Mais deux autres textes semblent suggérer une attitude moins passive. Le premier, après avoir expliqué que l'historien qui aspire à l'objectivité doit se faire une idée des exigences et des normes auxquelles il devrait se conformer, ajoute : "Mais son idée n'est et ne sera jamais qu'une hypothèse à confronter avec les réactions de tous ceux qui sont censés faire partie de l'auditoire universel auquel il s'adresse ${ }^{21} \gg$. Le second texte va dans le même sens, mais encore plus expressément :

Le recours à l'évidence donnait au rationalisme classique une assurance que le rationalisme thétorique ne possède plus, car ce qui pour celui-là se présentait comme une certitude ne peut constituer pour celui-ci qu'une hypothèse soumise à l'épreuve ; il ne suffit plus de présumer l'accord de l'auditoire universel, il faut s'en assurer effectivement ${ }^{22}$.

Le troisième trait de l'auditoire universel est étroitement lié à cette préoccupation de s'assurer effectivement de l'accord de l'auditoire universel. En effet, si, en tentant de vérifier son hypothèse, l'orateur devait découvrir que les thèses qu'il croyait acceptables par tous ne le sont pas en fait, quelle attitude devrait-il adopter? Sans doute pourra-t-il reconnaître qu'il s'est trompé et modifier sa conception de l'auditoire universel (texte 4), mais il pourra aussi maintenir sa conception de l'auditoire universel et, de celui-ci, exclure le

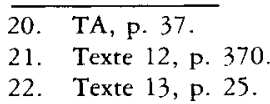


récalcitrant en prouvant qu'il est incompétent ${ }^{23}$ (textes 2-4TA-ÉTA-ER-LJ). Si l'orateur accroît ses exigences, il pourra même modifier la nature de son auditoire :

on voir comment, grâce à un pareil procédé, l'auditoire universel peut faire place à ce que je qualifierai d'auditoire d'élite, la qualité remplaçant la quantité. Ce sera l'auditoire qui ne comportera que les êtres normaux, les bons, les sages, les compétents, ceux qui ont la grâce, auxquels auront été révélées des vérités inaccessibles à l'ensemble des hommes ${ }^{24}$.

En résumé ${ }^{25}$, l'auditoire universel se compose de tous les êtres humains raisonnables (ou de tous les êtres humains raisonnables et compétents), et, comme il incarne la raison, on ne doit utiliser, en s'adressant à lui, que des arguments raisonnables ${ }^{26}$, c'est-à-dire universalisables. Cet auditoire n'est pas une réalité sociale concrète, mais une construction mentale que fait l'orateur à partir de sa propre expérience ; il est donc nécessairement variable, sans être pour autant arbitraire puisque, d'une part, il relève non des faits, mais du droit, et

23. Cette fois encore, le terme "incompétent " tient lieu d'une consteliation d'expressions comme "se tromper ", ou être "un monstre", " de parti pris", " de mauvaise volonté ", " anormal ", " déraisonnable », "insensé ", « stupide ». En général, la preuve de l'incompétence du récalcitrant incombe à l'orateur, mais, dans un texte (ER), la situation est inversée : c'est au récalcitrant à prouver qu'il n'est pas l'insensé qui s'oppose à l'opinion commune.

24. Texte 4. La même idée se retrouve dans le texte 2 et dans ÉTA, mais, dans le second cas, l'auditoire d'élite n'est pas nommé : si le récalcitrant, dit Perelman, est le porte-parole d'un grand nombre, voire de la majorité, l'orateur se référera progressivement non au sens commun, mais à des qualités spéciales exigées de ceux qui veulent accéder aux vérités qu'il défend, comme la grâce ou la compétence.

25. En trois textes (2-10-12), Perelman stipule que l'orateur doit être inclus dans l'auditoire universel. Nous ne retenons pas cette exigence pour trois raisons. Tout d'abord, si l'orateur est celui qui présente l'argumentation, et sí l'auditoire se compose de tous ceux que vise l'argumentation, il faudrait, pour admettre que l'orateur appartient à l'auditoire universel, supposer qu'il cherche à se convaincre lui-même. En second lieu, même si l'orateur est tenu de s'adapter à son auditoire, et partant d'argumenter à partir de prémisses admises par cet auditoire, il n'est pas toujours obligé d'admetzre lui-même ces prémisses, comme le montre l'exemple de l'argumentation ad bominem (sur celle-ci, cf. : TA, p. 148-150, et texte 10) ; et lorsqu'il les admet, il ne devient pas pour autant un auditeur. Troisièmement, on peut reconnaître que celui qui s'adresse à anus les êtres raisonnables doit être lui-même raisonnable, sans pour autant confondre ceux-ci avec celui-là.

26. À la distinction entre raison théorique ex raison prarique, Perelman en est venu à faire correspondre deux adjectifs : "rationnel " dans le premier cas, "raisonnable " dans le second (cf. "The Rational and the Reasonable ", in La rationalité aujourd'bui, éd. par Th. Geraets, Ottawa : Éditions de l'Université d'Ottawa, 1979, p. 213-224). Même si, en nombre de textes antérieurs, les deux adjectifs apparaissent comme interchangeables, il est préférable d'utiliser systématiquement le second en rapport avec l'argumentation. 
que, d'autre part, il équivaut à une hypothèse que l'orateur doit vérifier. Enfin, l'orateur peut exclure, de cet auditoire, tous ceux qui seraient incompétents, quitte à transformer l'auditoire universel en auditoire d'élite.

Le recours à l'auditoire universel ainsi défini implique-t-il une pétition de principe? Avant de répondre à cette question, nous devrons examiner deux problèmes dont la discussion permettra de préciser davantage à la fois la notion d'auditoire universel et l'enjeu du débat.

\subsection{Les problèmes que pose la notion d'auditoire universel}

Nous traiterons successivement de la relation entre l'auditoire universel et les autres types d'auditoire, du rapport entre le discours philosophique et d'autres types de discours, et de la pétition de principe impliquée ou non par le recours à l'auditoire universel.

\subsubsection{L'auditoire universel et les autres types d'auditoire}

Admettons pour le moment la thèse selon laquelle le recours à l'auditoire universel caractérise le discours philosophique : n'y a-t-il pas pourtant d'autres auditoires qui présentent un intérêt philosophique, en particulier la délibération intime, l'interlocuteur du dialogue, l'auditoire spécialisé et l'auditoire d'élite?

Le principe de la solution que propose Perelman se trouve dans l'affirmation que la distinction entre persuader et convaincre $^{27}$ dépend des intentions de l'orateur, qui peut n'envisager ceux auxquels il s'adresse, et ce même dans le cas de la délibération intime, qu'en tant qu'incarnation de l'auditoire universel ${ }^{28}$. Plus spécifiquement, on se rappellera que la délibération intime, discussion avec soi-même, a été pour l'essentiel ramenée au dialogue, discussion avec autrui; or, en certains cas, comme dans les dialogues platoniciens, l'auditeur unique incarne l'auditoire universel, ce qui explique l'intérêt philosophique de la forme dialoguée ${ }^{29}$. Que l'audi-

27. Convaincre, c'est obtenir l'adhésion de l'auditoire universel; qui se contente de l'adhésion d'un autre auditoire se borne à la persuasion (TA, p. 34-40; ER, p. 31).

28. ER, p. 31 .

29. TA, p. $47-48$ 
toire du philosophe ne constitue pas, par ailleurs, un auditoire spécialisé, c'est ce que Perelman affirme dans le Traité de l'argumentation $^{30}$ : tout philosophe s'adresse à l'auditoire universel, puisqu'il ne semble pas qu'en philosophie on puisse faire état d'un ensemble de connaissances, de règles et de techniques comparable au corps d'une discipline scientifique et commun à tous ceux qui pratiquent celle-ci. Quant à l'auditoire d'élite, Perelman déclare qu'il peut être considéré soit comme un groupe à part caractérisé par sa situation hiérarchique, soit comme un modèle dont seule compte l'opinion et auquel les autres hommes devraient se conformer ; or, en ce dernier cas, l'auditoire d'élite est assimilable à l'auditoire universel ${ }^{31}$.

Ces réponses sont-elles satisfaisantes? Nous les évaluerons en relation avec la problématique des divers types de discours, à laquelle elles sont étroitement liées, en conclusion de cet article.

\subsubsection{Le recours à l'auditoire universel est-il propre au discours philosopbique?}

En précisant que l'enjeu de la question relative à la pétition de principe est le discours philosophique lui-même, nous avons cité un texte dans lequel Perelman affirmait que le philosophe comme tel s'adresse à l'auditoire universel. Or, il importe de souligner qu'une telle déclaration n'est pas isolée : elle constitue au contraire un leitmotiv de la conception perelmanienne de la philosophie ${ }^{32}$. Sans doute tous les textes pertinents ne sont-ils pas également explicites, mais certains d'entre eux le sont suffisamment pour que l'on doive effectivement considérer l'auditoire universel comme spécifiant le discours philosophique ; ainsi :

C'est parce que l'effort du philosophe vise une espèce particulière d'auditoire que l'argumentation philosophique se distingue des autres argumentations rhétoriques ${ }^{33}$.

30. P. 134-135.

31. T.A. p. 44.

32. Ici encore, la fréquence des références est un bon indice. Pour nous en tenir à des textes que nous avons déjà utilisés, on pourra consulter les suivants: 2 (p. 38-39) -4-5-6-11-13-15-17-TA (p. 35-45)-ÉTA-ER.

33. Texte 4 , p. 126. 
C'est l'idéal philosophique par excellence de ne se servir - je crois qu'on pourrait même y trouver une définition de la philosophie - que d'arguments qui seraient conformes à l'impératif catégorique de Kant : il ne faudrait utiliser, dans un raisonnement philosophique, que des arguments qui pourraient valoir pour une universalité des esprits ${ }^{34}$.

On devrait donc s'attendre à ce que les autres types de discours different, à cet égard, du discours philosophique.

Tel est le cas du discours juridique. Celui-ci entretient, avec le discours philosophique, suffisamment de parenté pour qu'il puisse lui servir de modèle ${ }^{35}$, mais, en ce qui a trait à l'auditoire, il s'en distingue profondément : en effet, dans la mesure où il est lié par des textes bien déterminés, le droit positif constitue un domaine d'argumentation spécifique ${ }^{36}$, autrement dit, relève d'un auditoire spécialisé.

Il en va de même du discours religieux : contrairement au discours philosophique, il ne s'adresse pas à tous les êtres raisonnables, mais à un auditoire spécialisé qui admet des dogmes particuliers, non universalisables ${ }^{37}$.

Le discours de l'historien pose par contre un certain problème, car Perelman admet expressément qu'il puisse s'adresser à l'auditoire universel. On peut cependant résoudre ce problème en décrétant que, comme dans les cas précédents, l'historien devient alors philosophe, mais ce serait aller au-delà de ce que Perelman lui-même affirme:

Tout comme l'historien n'est jamais assuré d'être en possession de l'ensemble des sources relatives à certains éléments du passé, et qu'il n'a pas le droit d'écarter, sans raison, une source susceptible de l'éclairer, de même il n'a pas le droit d'écarter,

34. Texte 11, p. 300.

35. Perelman réaffirme sans cesse la pertinence du modèle juridique. Cf. en particulier "Ce qu'une réflexion sur le droit peut apporter au philosophe " (JR, p. 244-255) et "Ce que le philosophe peut apprendre par l'étude du droit » (DMP, p. 191-201).

36. TA, p. 135-136. À noter que le droit naturel n'est pas touché par cette affirmation; mais on pourrait arguer que si son élaboration fait appel à l'auditoire universel, il relève du domaine philosophique.

37. Cf. textes 13-15-16-TA-CL. L'un de ces textes (TA) fait exception pour la théologie rationnelle: mais, comme dans le cas du droit naturel, on pourrair arguer qu'elle appartient au domaine philosophique, ce que pourrait confirmer l'affirmation que le croyant qui tente de convaincre un incroyant doit argumenter philosophiquement (CL). Par ailleurs, un autre document (13) mentionne que le discours politique s'apparente au discours théologique en ce qu'il ne s'adresse pas à tous les hommes raisonnables. 
sans examen, une exigence que lui présenterait, en matière d'intelligibilité, un membre de cet auditoire universel, tel que le conçoivent les historiens. La discussion, sur ces matières, prendra bien souvent l'allure d'une discussion philosophique $(. . .)^{38}$

Prendre «l'allure d'une discussion philosophique », ce n'est pas devenir une discussion philosophique. Si donc les deux types de discours restent distincts même s'ils s'adressent tous deux à l'auditoire universel, il faudra, pour préserver la spécificité philosophique de l'auditoire universel, trouver d'autres arguments ; on pourrait, par exemple, en s'appuyant sur l'idée que ce ne sont que "les meilleurs historiens " qui aspirent à l'idéal d'objectivité représenté par l'auditoire universel $^{39}$, dire que celui-ci demeure la caractéristique propre du discours philosophique dans la mesure où le philosophe est contraint d'y recourir, alors que l'historien n'y est pas obligé. Mais une telle solution ne réglerait pas le problème que pose le discours scientifique.

En expliquant le caractère variable de l'auditoire universel, Perelman mentionne que chaque science a le sien (texte 2). Dans le Traité de l'argumentation, il écrit :

Certains auditoires spécialisés sont volontiers assimilés à l'auditoire universel, tel l'auditoire du savant s'adressant à ses pairs. Le savant s'adresse à certains hommes particulièrement compétents et qui admettent les données d'un système bien défini, constitué par la science dans laquelle ils sont spécialisés. Pourtant, cet auditoire si limité est généralement considéré par le savant non comme un auditoire particulier, mais comme étant vraiment l'auditoire universel : il suppose que tous les hommes, avec le même entraînement, la même compétence et la même information, adopteraient les mêmes conclusions ${ }^{40}$.

Perelman n'examine pas les problèmes que posent pareilles affirmations, mais nous allons le faire, car, si le savant s'adresse

38. Texte 12, p. 370 ; c'est moi qui souligne.

39. Ibid., p. 368. On trouve une restriction semblable dans un texte affirmant que l'auditoire universel est visé "dans les raisonnements les plus élevés de la philosophie "; ce texte ajoute même que les arguments qu'on analysera seront ceux que les esprits les plus droits " ne peuvent pas ne pas utiliser quand il s'agit de certaines matières, telle [sic !] que la philosophie et les sciences bumaines » (texte 2, p. 38-39; c'est moi qui souligne) ; ce texte, cependant, est ancien (1950) et a été amendé par plusieurs textes subséquents, ce qui n'est pas le cas du précédent.

40. TA, p. 44-45. 
lui aussi en principe à l'auditoire universel, comment ce dernier pourrait-il caractériser le discours philosophique?

On pourrait tenter d'escamoter le problème en posant en principe que la notion d'auditoire est étrangère au discours scientifique. Nous avons vu, en effet, que la logique au sens large du terme comprend d'une part la logique formelle, d'autre part la rhétorique; si donc la notion d'auditoire est propre à la rhétorique, et si l'activité scientifique relève plutôt de la logique formelle, ne faudrait-il pas conclure qu'effectivement la science n'est pas relative à un auditoire ${ }^{41}$ ? Cette conclusion, cependant, reposerait sur une confusion relative à la notion d'auditoire. Selon la nouvelle rhétorique, en effet, l'auditoire se compose de tous ceux que vise l'argumentation : mais n'est-ce pas trop limiter cette notion que de ne la définir qu'en fonction de l'argumentation? La démonstration ne s'adresse-t-elle pas, elle aussi, à quelqu'un? Pour lever l'ambiguïté sans modifier le sens perelmanien de la notion d'auditoire, empruntons à la sémiologie de la communication le concept bien connu de destinataire et reconnaissons que tout raisonnement, qu'il s'agisse d'une démonstration ou d'une argumentation, s'adresse à un destinataire : on pourra dès lors appeler auditoire tout ensemble de destinataires dont le destinateur doit connaître les opinions pour appuyer sur elles son raisonnement, ou, plus brièvement, le destinataire d'une argumentation. On pourra ainsi affirmer, sans confondre le fait de s'adresser à un auditoire et le fait de tenir compte de ce qu'il admet, que le savant s'adresse à des destinataires, mais non à des auditeurs. Affirmation qui fera cependant surgir deux nouveaux problèmes. Le premier est lié à la conception perelmanienne de l'activité scientifique : ceux qui réduisent la science à un enchaînement impersonnel de propositions en oublient l'aspect créateur (v.g. l'invention des hypothèses), qui

41. Cette argumentation s'appuie (en en étendant la portée) sur le texte suivant : «Contrairement à une démonstration mathématique, toute argumentation s'adresse à un auditoire et est nettement conditionnée par la nature de celui-ci. Alors que la démonstration se présente comme impersonnelle, le discours argumentatif est toujours situé " (ÉTA, p. 17). Dans un autre texte, après avoir posé la distinction entre démonstration et argumentation, et après avoir affirmé que la première échappe au conditionnement social, Perelman ajoute: "toute argumentation se développe en fonction de l'auditoire auquel elle s'adresse et auquel l'orateur est obligé de s'adapter * (texte 9, p. 24). Voir aussi le texte 16. 
oblige le savant à s'engager et à prendre ses responsabilités ; or, par ce biais, la nouvelle rhétorique s'introduit au cœur même des débats théoriques ${ }^{42}$. Il n'en reste pas moins, entre la science et les autres domaines de la culture, une différence fondamentale :

C'est un fait indéniable dans l'histoire des sciences que, à chaque époque, il existe un ensemble de thèses sur lesquelles la communauté scientifique est d'accord jusqu'à nouvel ordre. Même si on en parle avec mépris comme de la science morte, même si l'on n'est pas entièrement d'accord sur l'énumération de ces vérités, même s'il ne s'agit que de vérités temporaires dont plusieurs seront remises en question à l'occasion d'une grande découverte ultérieure, l'existence d'un corpus de vérités admises constitue une réalité dont l'importance ne peut être négligée. On pourra éventuellement s'opposer à l'une ou l'autre de ces propositions, mais à condition de montrer leur incompatibilité avec d'autres propositions plus solidement établies; il faudra, en tout cas, les présumer vraies jusqu'à preuve du contraite. Or il faut bien constater qu'un pareil corpus des vérités admises n'existe ni en philosophie, ni en droit, ni dans le domaine artistique, moral ou religieux, du moins quand on dépasse les bornes d'une communauté particulière ${ }^{4.3}$.

Ces remarques permettent de formuler le second problème. Le corpus des vérités admises devrait en effet constituer les savants en auditoire spécialisé : mais comment peut-on accepter d'utiliser ici cette notion si la science, en tant que système de propositions et non en tant qu'activité heuristique, est impersonnelle et n'admet pas la référence à un auditoire ? Ce problème est d'autant plus complexe que, comme nous l'avons constaté plus haut, cet auditoire spécialisé peut être considéré comme un auditoire universel. Pour le résoudre, nous pourrions maintenir, à l'encontre de Perelman, que la notion d'auditoire n'est pas pertinente dans le contexte des vérités scientifiques. Mais comme Perelman soutient que l'auditoire universel constitue l'incarnation de la raison, le remède risquerait d'être pire que le mal, car il transformerait la science

42. À ce sujet, cf. "La quête du rationnel " (RP, p. 110-120); "Méthodologie scientifique et philosophie ouverte" (in Revue internationale de pbilosopbie, 93-94 (1970), p. 623-628); "La conception de la recherche scientifique de M. Polanyi (CA, p. 344-352); ER (p. 22).

43. "La conception de la techerche scientifique de M. Polanyi ", p. 349 
en activité déraisonnable. La recherche d'une autre solution s'impose donc, et à cette fin nous allons revenir à la notion d'auditoire et à celle de pétition de principe.

\subsubsection{Le recours à l'auditoire universel implique-t-il une pétition de principe?}

Nous venons de constater que le discours scientifique se caractérise par un corpus de vérités admises, c'est-à-dire de propositions qui doivent être présumées vraies jusqu'à preuve du contraire. Or, cette notion de présomption est aussi utilisée par Perelman en relation avec celle d'auditoire universel : le raisonnable, c'est "l'adbésion présumée de tous ceux que l'on considère comme des interlocuteurs valables concernant les questions débattues ${ }^{44}$ "; d'ailleurs, si l'on considère non le mot, mais la chose, on admettra que la définition même de l'auditoire universel implique une présomption, puisque l'accord universel que l'on souhaite n'est jamais donné effectivement mais est imaginé par l'orateur. Or, supposer admise une prémisse qui ne l'est pas, n'est-ce pas la définition même de la pétition de principe?

Dans le Traité de l'argumentation ${ }^{45}$, le problème de la pétition de principe est abordé dans le cadre d'une discussion de l'argument ad bominem. Perelman pose d'abord que les possibilités d'argumentation dépendent de ce que chacun est disposé à concéder et qu'en ce sens toute argumentation est ad bominem ou ex concessis; par suite, ceux qui opposent à l'argumentation ad bominem, portant sur l'opinion, l'argumentation ad rem, relative à la vérité ou à la chose même, oublient que cette vérité doit être admise : l'argumentation ad rem est une argumentation que l'on prétend valable pour toute l'humanité raisonnable, c'est une argumentation ad bumanitatem, une argumentation visant l'auditoire universel, et elle est un cas particulier de l'argumentation ad bominem ${ }^{46}$. Or, c'est par rapport à celle-ci que l'on peut comprendre en quoi consiste la pétition de principe :

44. Texte 9, p. 72 ; c'est moi qui souligne.

45. TA, p. 148-153.

46. "Il ne faut pas confondre l'argument ad bominem avec largument ad personam, c'est-à-dire avec une attaque contre la personne de l'adversaire et qui vise, essentiellement, à disqualifier ce dernier " (TA, p. 150). 
La pétition de principe consiste en un usage de l'argument ad bominem lorsqu'il n'est pas utilisable, parce qu'elle suppose que l'interlocuteur a déjà adhéré à une thèse que l'on s'efforce justement de lui faire admettre. Encore faut-il que les deux propositions, le principe et la conclusion, qui ne sont jamais exactement les mêmes, soient suffisamment proches l'une de l'autre pour que l'accusation de pétition de principe soit justifiée. Aussi une discussion surgit-elle presque toujours au sujet du bien-fondé de l'accusation.

Et, comme nous l'avons noté plus haut, il faudra que la prémisse contestée n'ait aucun autre fondement que la conclusion qu'on a voulu en tirer et pour laquelle elle constitue un chaînon indispensable dans le raisonnement. Perelman donne en exemple ce passage d'un discours d'Antiphon :

Sachez bien que je mérite votre pitié beaucoup plus qu'un châtiment. Le châtiment revient, en effet, aux coupables, la pitié à ceux qui sont l'objet d'une accusation injuste.

Il le commente comme suit :

L'ordre de la majeure et de la conclusion est inversé. La mineure sous-entendue "Je suis l'objet d'une accusation injuste" ne peut être admise par les auditeurs parce que, si elle avait été accordée, le procès serait jugé. C'est la raison pour laquelle Antiphon, au lieu de présenter le droit qu'il prétend avoir à la pitié comme la conclusion d'un syllogisme, présente son affirmation avant la majeure, pour lui donner une sorte de validité indépendante.

Peut-on soutenir que le recours à l'auditoire universel présuppose une telle adhésion à des prémisses qu'il s'agit de faire admettre? Oui, si l'on reconnaît que la pétition de principe peut être potentielle. Rappelons-nous, en effet, que l'auditoire universel se caractérise par trois traits principaux : a) il se compose de l'ensemble des êtres humains raisonnables (et compétents) ; b) cet ensemble est fictif, il équivaut à une hypothèse ; c) celle-ci doit être vérifiée. Rappelons-nous aussi la thèse stipulant que l'orateur doit s'adapter à. son auditoire, c'est-à-dire partir de prémisses admises par celui-ci (argumentation ad bominem). Supposons maintenant qu'un orateur donné a décidé de s'adresser à l'auditoire universel. Par définition, cet orateur ne peut connaître toutes les prémisses admises, ni même une seule prémisse admise par l'ensemble des êtres 
humains raisonnables (et compétents), puisque, si tel était le cas, son auditoire cesserait d'être fictif. Notre orateur ne peut donc que présumer que les prémisses qu'il suppose admises le sont effectivement. Mais s'il savait qu'elles ne le sont pas, il devrait soit les abandonner, soit les faire admettre par ses auditeurs : ces prémisses sont donc potentiellement contestables. Il s'ensuit que le recours à l'auditoire universel implique sinon une pétition de principe effective, du moins une pétition de principe potentielle. Sans doute pourrait-on objecter que cette distinction a peu de poids et que toute argumentation repose sur une pétition de principe au moins potentielle; mais ce serait oublier que, dans le cas de l'auditoire spécialisé, l'orateur connaît les prémisses qu'admettent ses auditeurs, tandis que, dans le cas du dialogue, il doit s'assurer à chaque étape de l'accord de son interlocuteur : ce n'est donc que par rapport à l'auditoire universel que la notion de pétition de principe potentielle se révèle utile. Objectera-ton, alors, que dans la mesure où l'auditoire universel est une construction de l'orateur, il admet nécessairement les prémisses que lui impute ce dernier, et que ce n'est que par rapport à un auditoire réel qu'il pourrait être question de pétition de principe potentielle? Si l'on s'en tenait aux deux premiers traits qui définissent l'auditoire universel, cette objection serait valable, mais en y faisant droit ou bien la théorie deviendrait inconsistante, puisque la définition de l'auditoire universel serait incompatible avec l'obligation imposée à l'orateur de partir de prémisses admises par ses auditeurs, ou bien elle deviendrait ambiguë, puisque l'expression "prémisses admises par l'auditoire » concernerait tantôt des auditoires réels, tantôt des auditoires fictifs ; pour éviter ce double inconvénient, il faut effectivement recourir à un auditoire réel : mais n'est-ce pas précisément ce à quoi nous invite le troisième trait qui définit l'auditoire universel, en décrétant que l'hypothèse à laquelle équivaut cet auditoire doit être vérifiée ? Ce n'est que par rapport à des auditeurs réels que la pétition de principe potentielle se transformera ou non en pétition de principe réelle, selon que ceux-ci admettront effectivement ou non les prémisses utilisées par l'orateur. Mais on pourrait encore objecter que, dans toute la discussion précédente, la pétition de principe a été assimilée au simple fait d'utiliser une 
prémisse comme si elle était admise alors qu'elle ne l'est peut-être pas, sans tenir compte des autres conditions nécessaires à l'existence d'une pétition de principe, à savoir que l'auditeur conteste la prémisse en question parce qu'elle postule une proposition qu'il s'agissait de prouver ou de faire admettre, que le principe et la conclusion soient suffisamment rapprochés pour que l'accusation soit justifiée, et que la prémisse contestée n'ait pas d'autre fondement que la conclusion qu'on a voulu en tirer. À cela l'on peut répondre que les conditions d'existence d'une pérition de principe potentielle ne sont pas les mêmes que celles d'une pétition de principe effective. Par ailleurs, on peut constater que Perelman luimême, à la fin du passage auquel nous nous sommes référé, utilise la notion de pétition de principe d'une façon très large :

Pour conclure, la pétition de principe est une faute d'argumentation. Elle concerne l'argumentation ad hominem et la présuppose, car son domaine n'est pas celui de la vérité mais celui de l'adhésion. Si l'on reconnaît qu'il est illégitime de commettre des pétitions de principes, c'est-à-dire de fonder son argumentation sur des prémisses que l'auditoire rejette [c'est moi qui souligne], cela implique que l'on peut se servir de celles qu'il admet. Quand il s'agit de vérité, et non d'adhésion, l'argument ad hominem est à proscrire, mais dans ce cas la pétition de principe est impossible. Les deux sont corrélatifs : on ne peut tenir compte de l'accusation de "pétition de principe » et peser la légitimité de la critique qu'elle implique que dans le cadre d'une théorie de l'argumentation.

\section{Conclusion}

Si le discours philosophique s'adresse à l'auditoire universel, il implique donc une pétition de principe potentielle. En pratique, les propositions philosophiques assimilables à une telle pétition de principe devraient coïncider avec les premiers principes d'une philosophie. Cela signifie que le philosophe, lorsqu'il pose de tels principes, souhaite qu'ils soient admis par tout être de raison, mais en sachant fort bien qu'ils seront très probablement contestés ${ }^{47}$ et qu'alors il lui incombera de les

47. Si personnel que soit son système, le philosophe doit au moins avoir appris, de l'histoire de sa discipline, qu'elle ne comporte guère de propositions qui soient universellement admises. 
justifier. Cette conception des premiers principes comme n'étant que provisoirement premiers est tout à fait conforme à la position de Perelman:

ces principes métaphysiques constituent, à mes yeux, non un point de départ inévitable de toute philosophie, mais un point d'aboutissement dans l'analyse des présupposés de chacune d'entre elles. Ce point d'aboutissement, on y est mené par la recherche de thèses dont on croit qu'elles susciteraient l'accord de l'auditoire universel, constitué par tous les hommes raisonnables et compétents en la matière.

L'analyse régressive qui mène à ces principes n'aboutit donc pas à des thèses qui seraient, elles, sans présupposé d'aucune sorte, car cela supposerait, ce qui me semble absurde, la possibilité d'évidences indépendantes de tout contexte. Les principes métaphysiques auxquels on s'arrête, pour y voir les fondements du système, sont des principes qui, dans leur contexte, paraissent suffisamment assurés au philosophe pour qu'il puisse les présenter comme tels à tout être qu'il considère comme raisonnable. Mais ce qui paraît assuré et mérite le nom de premier principe, pour un philosophe, peut nécessiter un approfondissement, un recul vers d'autres principes, pour un autre philosophe, spécialement s'il vit dans un autre contexte historique, culturel et social ${ }^{48}$.

Provisoires, les premiers principes sont dès lors soumis à un "principe de révisibilité » dont il importe cependant de constater qu'il « ne pose qu'une possibilité de réviser certaines affirmations, si se présentent des raisons impérieuses de le faire ${ }^{49} "$.

En plus d'être conforme à la conception perelmanienne de la métaphysique, notre interprétation permet de résoudre les principaux problèmes liés à la notion d'auditoire universel.

Commençons par la définition de cet auditoire. Selon Perelman, le philosophe peut disqualifier les récalcitrants en les considérant comme incompétents, stupides, déraisonnables, etc. Mais une telle justification de la disqualification constitue un argument ad personam, lequel est incompatible avec l'obligation imposée au philosophe de n'utiliser que des arguments valables pour l'auditoire universel. Pour lever cette

48. "Réponse à une enquête sur la métaphysique " (in CA, p. 251).

49. "Philosophies premières et philosophie régressive" (in RP, p. 98). 
incompatibilité, il faudrait admettre que le philosophe n'a le droit de disqualifier aucun interlocuteur, que tout être raisonnable est, en tant que tel, compétent en philosophie ${ }^{50}$. En admettant cette solution, on peut résoudre également le problème de la double description de la composition de l'auditoire universel, qui regroupe tantôt tous les êtres humains raisonnables, tantôt tous les êtres humains raisonnables et compétents : notre solution au problème précédent nous incite en effet à considérer ces deux adjectifs comme synonymes en l'occurrence ${ }^{51}$, ce qui nous laisse une seule description. L'auditoire universel se compose donc de tous les êtres humains raisonnables, et, en tant que tels, ceux-ci sont compétents en matière philosophique, si bien qu'aucun d'eux ne peut être disqualifié à cet égard. Mais cela n'implique pas que le philosophe soit à la merci de ses auditeurs. Dans une conception rhétorique de la philosophie, le discours philosophique se ramène en effet à une argumentation: mais argumenter, c'est donner des raisons pour ou contre une thèse, et cette exigence s'applique non seulement à l'orateur, mais aussi à l'auditeur qui conteste son discours. Est raisonnable toute objection fondée sur des raisons, et le philosophe se doit de tenir compte des objections raisonnables.

Revenons maintenant à la relation entre l'auditoire universel et les autres types d'auditoires, en particulier l'auditoire d'élite et l'auditoire spécialisé. Déjà une incompatibilité existait entre les textes de Perelman qui utilisaient la notion de compétence pour caractériser et l'auditoire universel, et l'auditoire d'élite ; mais si l'on admet que les membres de l'auditoire universel sont par définition compétents, celui-ci devient radicalement incompatible avec l'auditoire d'élite.

50. Cette décision est elle aussi conforme à la pensée de Perelman, ou plutôt à un aspect de sa pensée qui ne s'est exprimé comme tel, à notre connaissance, qu'une fois : "Ce qui caractérise le discours philosophique, par opposition au discours théologique, qui ne s'adresse qu'aux croyants admettant au départ certains dogmes ou certains textes sacrés, et au discours politique, qui ne vise qu'une communauté particulière, ayant des valeurs et des aspirations qui lui sont propres, c'est qu'il vise tous les bommes vaisonnables, et que chacun de ceux-ci est donc en droit de le contester" (rexte 13, p. 26 ; c'est moi qui souligne).

51. Que les deux termes ne soient pas, pour Perelman, synonymes, on peut le constater dans une formulation comme celle-ci : "l'auditoire universel [est] cornposé de tous ceux qui sont disposés à l'entendre, et qui sont capables de suivre son argumentation " (ER, p. 30) ; la dernière partie de la formule peut être considérée comme décrivant la compétence, mais non la rationalité. 
Nous suggérons de considérer l'auditoire d'élite comme un auditoire non philosophique qui résulte du passage de la pétition de principe potentielle à la pétition de principe effective. Le récalcitrant, en effet, doit, pour devoir être écouté, motiver ses objections : or, ou bien le philosophe peut réfuter ces motifs, et alors il n'a pas besoin de recourir à l'auditoire d'élite pour maintenir ses positions, ou bien il ne peut réfuter les raisons alléguées, et, en pareil cas, s'il maintient ses thèses, c'est en renonçant à convaincre l'auditoire universel. Le recours à l'auditoire d'élite devient ainsi l'indice d'une démission philosophique. Le problème de l'auditoire spécialisé est plus complexe. La raison pour laquelle Perelman refuse de considérer que le discours philosophique s'adresse à un auditoire spécialisé, c'est que, contrairement à ce qu'il se passe en science, il n'existe pas, en philosophie, un corpus de "vérités» qui seraient universellement admises. En d'autres termes, si les "vérités" sont universellement admises, l'auditoire est particulier, mais si les "vérités"sont particulières, l'auditoire est universel! Mais s'il en était vraiment ainsi, comment expliquer le fait que pour Perelman le discours religieux s'adresse à un auditoire spécialisé, alors qu'il n'existe manifestement pas de «vérités » religieuses universellement admises? Dans "Philosophie et argumentation " (texte 10), en réponse à la thèse de Johnstone à l'effet que le désaccord entre les philosophes empêche que leur auditoire soit universel et qu'en fait leur argumentation s'adresse à des auditoires particuliers liés par des obligations qui leur sont propres, Perelman rétorque qu'il est vrai que peu d'affirmations philosophiques sont universellement admises, mais que les prémisses à propos desquelles on peut espérer un tel accord relèvent non de la philosophie mais du sens commun, de l'histoire, de la science, et qu'une argumentation rationnelle peut aussi partir de prémisses admises par certains et en tirer des conclusions admises par tous. Pourtant, en ce dernier cas, les prémisses ne seraient-elles pas ad bominem sans être ad bumanitatem? À propos de l'usage de l'argument ad bominem « consistant à mettre l'interlocuteur en contradiction avec ses propres affirmations, avec les enseignements d'un parti qu'il approuve ou avec ses propres actes ", Perelman fait observer qu'il « n'y a rien d'illégitime dans cette façon de procéder » et 
qu'on pourrait même «qualifier pareille argumentation de rationnelle, tout en admettant que les prémisses discutées ne sont pas admises par tous $^{52} »$. Perelman n'examine pas l'incompatibilité entre cette suggestion et l'affirmation réitérée que le philosophe doit partir de prémisses valables pour tous. Pourtant, cette incompatibilité est d'autant plus gênante que si l'on assimile l'usage de la raison à l'appel à l'auditoire universel, la majorité des débats philosophiques risquent de devenir irrationnels. En effet, dans la pratique philosophique, c'est moins un philosophe qui discute avec un autre philosophe que, disons, un marxiste qui discute avec un autre marxiste (et ils s'accordent sur les prémisses) ou avec un phénoménologue (et ils ne s'accordent pas sur les prémisses, quoique rien n'empêche l'un de partir des prémisses de l'autre) : mais ces formes d'argumentation sont-elles ou non raisonnables? On peut soutenir qu'elles le sont, comme Perelman l'a fait sans s'expliquer à ce sujet, en marquant bien la distinction entre auditoire concret et auditoire idéal : dans les cas auxquels nous venons de faire allusion en nous inspirant de l'objection de Johnstone, on peut en effet soutenir que l'auditeur concret peut être considéré comme incarnant l'auditoire universel si l'on reconnaît que tout homme raisonnable qui admettrait telles prémisses (marxistes ou phénoménologiques, pour reprendre nos exemples) devrait aussi admettre telle conclusion. On peut maintenant comprendre comment l'auditoire du philosophe peut être à la fois particulier et universel. On peut aussi constater que ce corpus de «vérités» admises qui caractérise la science se retrouve non au niveau de la philosophie en général, considérée abstraitement, mais au niveau des philosophies particulières ${ }^{53}$. Cette constatation nous oblige à dire un mot, pour terminer, du rapport entre l'auditoire universel et les divers types de discours.

La science, telle que la décrit Perelman, ne regroupe pas une pluralité d'écoles; elle comporte un corpus de "vérités" universellement admises et s'adresse à un auditoire spécialisé

52. TA, p. 149:

53. Ce pluralisme de la philosophie est un autre des leitmotive de la pensée de Perelman qui écrit, par exemple, qu'il faut "tenir compre du fait indéniable, celui de la pluralité de philosophies irréductibles". ("Méthodologie scientifique et philosophie ouverte", p. 626). 
qui peut être considéré comme une incarnation de l'auditoire universel. Le discours philosophique, qui s'adresse à l'auditoire universel, comprend une pluralité d'écoles et ne comporte pas de corpus de "vérités» admises. Quant au discours religieux, qui s'adresse à un auditoire spécialisé, il comprend une pluralité de sectes dont chacune admet certains dogmes et certains textes sacrés. La comparaison, comme on peut le constater, est en porte-à-faux. En décrivant globalement le discours philosophique, Perelman occulte les thèses admises par chaque école, tandis qu'en décrivant particulièrement les sectes religieuses, il néglige le fait qu'à un niveau plus général il n'y a guère de dogmes communs à toutes les religions. Mais dès que l'on prend en considération ces données du problème, la ressemblance entre le discours religieux et le discours philosophique devient telle qu'on peut se demander ce qui les distingue. La différence, à notre avis, se situe, d'une part, au niveau des thèses propres aux écoles ou aux sectes : elles seraient universalisables en philosophie, mais non en théologie; ainsi qu'au niveau des principes, qui seraient révisables en philosophie, mais non en religion : du point de vue de l'auditoire universel, le dogme est une pétition de principe effective ${ }^{54}$. Quant au discours scientifique, deux solutions sont possibles à son égard. La première consisterait à reprendre la suggestion que nous faisions plus haut et à considérer que la notion d'auditoire n'est pas pertinente dans le cas du discours scientifique, celui-ci se distinguant alors du discours philosophique, négativement, par le fait de n'être pas adressé à un auditoire, et, positivement, par un autre critère, tel celui de vérité ; c'est cette solution que semble préconiser le texte suivant :

L'existence d'une pluralité de philosophies différentes nous incite à croire que le propos du philosophe, contrairement à celui du savant spécialisé, n'est pas de nous faire connaitre la vérité sur un objet bien défini, mais de présenter à tous les hommes une vision du monde raisonnable ${ }^{55}$.

54. Le discours politique est assimilable au discours religieux: qui n'accepte pas les principes de base d'un parti n'a quà changer d'option.

55. "Philosophie et rhétorique ", in Tijdschrift voor Filosofie, p. 441-442; cf. également: texte 13 , p. 26, et texte 15 , p. 191-192. 
La seconde solution consisterait à relativiser au maximum la notion de science à partir de l'idée que le corpus des vérités admises n'est pas éternel et, en reprenant l'affirmation que son auditoire spécialisé incarne l'auditoire universel, à admettre qu'elle s'adresse elle aussi à l'auditoire universel, et même de façon privilégiée, puisqu'elle jouit, en dehors des périodes de crise, d'un accord unanime. Avéc cette seconde solution, l'auditoire universel, et par conséquent l'appel à la raison, ne serait plus l'apanage du discours philosophique, lequel occuperait une position intermédiaire entre discours scientifique et discours religieux. Si l'une et l'autre solution se révélaient inadéquates, il faudrait soit renoncer à la notion d'auditoire universel, soit reconnaitre que le philosophe ne s'adresse qu'à une fiction. Ce qui expliqueraient peut-être pourquoi il est si rarement entendu.

Faculté de philosophie

Université Laval 